\title{
Derivation of turbulent energy in presence of dust particles
}

\author{
Shams Forruque Ahmed \\ Senior Lecturer in Mathematics, Prime University, Dhaka, Bangladesh
}

Email address:

Ahmed_jhe99@yahoo.com

\section{To cite this article:}

Shams Forruque Ahmed. Derivation of Turbulent Energy in Presence of Dust Particles. American Journal of Applied Mathematics. Vol. 1, No. 4, 2013, pp. 71-77. doi: 10.11648/j.ajam.20130104.15

\begin{abstract}
Energy equation for dusty fluid turbulent flow has been derived in terms of correlation tensors of second order. In presence of dust particles, mathematical modeling of turbulent energy is discussed including the correlation between the pressure fluctuations and velocity fluctuations at two points of the flow field, where the correlation tensors are the functions of space coordinates, distance between two points and time. To reveal the relation of turbulent energy between the two points, one point has been taken as origin of the coordinate system.
\end{abstract}

Keywords: Energy Equation, Turbulent Flow, Dust Particle, Two-point Correlation, Correlation Tensor

\section{Introduction}

In a turbulent fluid the behavior of dust particles depend on the concentration of the particles and on the size of the particles with respect to the scale of turbulent fluid. The turbulent flow can be found in many areas of industry, such as the production of the composite materials, environmental engineering, chemical engineering, textile industry, paper making and so on. At great concentration there is an interaction between the particles through collisions and through the effects on the flow of the fluid in the neighborhood of the particles. Bracco et al. [4] described a scenario in which turbulence mediates a process by aggregating particles into anti-cyclonic regions. The simulation result of this study suggested that the anti-cyclonic vortices form as long-lived coherent structures, the process becomes more powerful because such vortices trap particles effectively. Turbulence driven by magneto rotational instability crucially affects the evolution of solid bodies in protoplanetary disks. The vertical structure of turbulence was found as well characterized by the vertical magnetic flux and three critical heights derived from the linear analysis of magneto rotational instability in a stratified disk [16].

Turbulence is maintained by the turbulent energy production, where the dissipation and the buoyancy flux act as sinks for the turbulent energy. Numerical models for turbulent fluid-particle flows were reviewed by Crowe et al. [6]. The review was structured according to the turbulence models used for the continuous phase: turbulence energy dissipation models, large-eddy simulations, direct numerical simulations, and discrete vortex models. Oakey [15] examined the rate of dissipation of turbulent energy from simultaneous temperature and velocity shear microstructure measurements. Spectra of turbulence were examined for both temperature gradient and velocity shear. Saito and Saito and Lemos [19] derived a macroscopic two-energy equation model for turbulent flow in a highly porous medium and applied to a porous channel bounded by parallel plates Macroscopic continuity, momentum and energy equations are presented local non-thermal equilibrium is considered by means of independent equations for the solid matrix and the working fluid.

Pan et al. [18] studied the clustering of inertial particles in turbulent flows and discussed its applications to dust particles in protoplanetary disks. Radial distribution function was computed using numerical simulations, which measures the probability of finding particle pairs at given distances, and the probability density function of the particle concentration. It was found that the particles of different sizes tend to cluster at different locations, leading to flat radial distribution functions between different particles at small scales. Johansen et al. [11] considered vertical plane of a protoplanetary disk including the Coriolis force and the radial advection of the Keplerian rotation flow. Dust grains, treated as individual particles, move under the influence of friction with the gas, while the gas was treated as a compressible fluid. The friction force from the dust grains on 
the gas leads to a vertical shear in the gas rotation velocity. As the particles settle around the midplane due to gravity, the shear increases, and eventually the flow becomes unstable to the Kelvin-Helmholtz instability. Hussainov et al. [10] explored experimental results dealing with the modulation of grid-generated turbulence by coarse glass particles in a vertical downward channel flow. Distributions of the mean velocity, the turbulence intensity along the channel axis and in different cross-sections obtained by Laser Doppler Anemometer were also presented. Bosse [3] investigated the gravitational settling of an initially random suspension of small solid particles in homogeneous turbulence. Results were presented for both one-way coupling, when the fluid flow is not affected by the presence of the particles, and two-way coupling, when the particles exert a feedback force on the fluid. For light particles the turbulence leads to a rapid redistribution of particles such that their density per unit mass is approximately constant with height. Hodgson and Brandenburg [9] investigated the formation of planetesimals by studying the transport of dust particles in a local three dimensional simulation of accretion disc turbulence. The polar mesospheric winter echoes dust particles that influence the radar backscatter most likely have sizes around 3 nanometer. Havnes and Kassa [7] discussed the charging of nanometer-sized particles and found that the photo detachment effect, where photons of energy less than the work function of the dust material can remove excess electrons, probably is dominant at sunlit conditions. Kvasnak et al. [13] designed, constructed, and used an experimental set-up to study the wall deposition rate of particles in a turbulent channel flow. The deposition rate of spherical particles was found to increase with diameter. The deposition velocities for compact dust components were found to closely resemble those of equivalent spherical particles.

Nickovic et al. [14] developed an integrated modeling system to describe the dust cycle accurately in the atmosphere. The dust modules of the entire system incorporated the state of the art parameterizations of all major phases of the atmospheric dust life such as production, diffusion, advection, and removal. These modules also included the effect of the particle size distribution on aerosol dispersion. Direct determination of the irreversible turbulent flux of salinity in the ocean has not been possible because of the complexity of measuring salinity on the smallest scales over which it mixes. Kishore and Sarker [12] determined the rate of change of vorticity covariance in magneto hydrodynamic turbulent flow of dusty incompressible fluid. The influence of dust particles on the turbulence in a vertical pipe at moderate particle concentrations was studied by Pakhomov et al. [17]. The radial profiles of the axial and radial velocity components and of the turbulent kinetic energy of the carrier (gas) and the dispersed phases were measured using two-component laser Doppler anemometer.

Hinze [8] derived an expression for turbulent motion in terms of correlation tensor of second order at two points of the flow field, where the correlation tensors were the functions of space coordinates, distance between two points and time. Chandrasekhar [5] introduced an independent variable in order to differentiate between the effects of distance and location. In the limiting case of zero viscosity and infinite electrical conductivity there exist two distinct modes of turbulence were shown; these had been distinguished as the velocity mode and the magnetic mode respectively. Ahmed and Sarker [2] derived an equation for turbulent fiber motion in terms of second order correlation tensor, where the correlation tensors were the functions of space coordinates, distance between two points and time. In presence of dust particles, they derived another equation of turbulent fiber motion in terms of second order correlation tensor [20]. Ahmed [1] also derived another equation in terms of second order correlation tensor for the derivation of turbulent energy in a rotating frame. However, there are few studies relevant to the dusty fluid turbulent energy although it is prevalent in the industry. In view of all these works, the main aim of the study is to derive an energy equation for dusty fluid turbulent flow at two pints of the flow field in terms of correlation tensor of second order, where the correlation tensors are the functions of space coordinates, distance between two points and time.

\section{Mathematical Model of the Problem}

Let us assume that the fluid is incompressible. The energy equations of motion and continuity for turbulent flow of a viscous incompressible fluid are:

$$
\begin{gathered}
\frac{\partial u_{i}}{\partial t}+u_{j} \frac{\partial u_{i}}{\partial x_{j}}=-\frac{1}{\rho} \frac{\partial p}{\partial x_{i}}+v \frac{\partial^{2} u_{i}}{\partial x_{j} \partial x_{j}}-2 \varepsilon_{i j l} \Omega_{j} u_{l} \\
\frac{\partial u_{i}}{\partial x_{i}}=0
\end{gathered}
$$

In presence of dust particles, the equations of motion are given by

$$
\begin{aligned}
& \frac{\partial u_{i}}{\partial t}+u_{j} \frac{\partial u_{i}}{\partial x_{j}}=-\frac{1}{\rho} \frac{\partial p}{\partial x_{i}}+v \frac{\partial^{2} u_{i}}{\partial x_{j} \partial x_{j}} \\
&-2 \varepsilon_{i j l} \Omega_{j} u_{l}+\frac{K N}{\rho}\left(v_{i}-u_{i}\right) \\
& \frac{\partial u_{i}}{\partial x_{i}}=0 \\
& \frac{\partial v_{i}}{\partial t}+v_{j} \frac{\partial u_{i}}{\partial x_{j}}=-\frac{K}{m_{s}}\left(u_{i}-v_{i}\right)
\end{aligned}
$$

where $u_{i}(x, t)$ is the fluid velocity components; $p$ is the unknown pressure field; $v$ is the kinematical viscosity of 
the fluid; $\rho$ is the density of the fluid particle; $\varepsilon_{i j l}$, the three-dimensional permutation symbol, where $\varepsilon$ is the dissipation by turbulence per unit of mass; $\Omega_{j}$, the rotation vector; $v_{i}(x, t)$, the solid particles (dust) velocity components; $p(x, t)$, the unknown pressure field; $m_{s}=4 \pi R_{s}^{3} \rho_{s} / 3$, the mass of a single spherical dust particles of radius $R_{s} ; v=$ constant is the molecular kinematical viscosity; $K=6 \pi R_{s} \rho v$, the Stoke's drag formula; $N$, the number density of dust particles; $K N / \rho=f$, has dimension of frequency and $\mathrm{t}$ is the time.

Let $A$ and $B$ be two points in the flow field, and let $a$ and $b$ be two given directions at the points $A$ and $B$ respectively, where $U_{a}$ and $U_{b}$ are the velocity components along these directions. We assume that the mean velocity $\overline{U_{i}}$ is constant throughout the region considered and independent of time, and we put

$$
\left(U_{i}=\bar{U}_{i}+u_{i}\right)_{A},\left(U_{j}=\bar{U}_{j}+u_{j}\right)_{B} .
$$

The value of each term can be obtained by using the equations of motion for $u_{j}$ at the point $B$ and for $u_{i}$ at the point $A$. The energy equation of motion for $u_{i}$ at the point $A$ is obtained from Equation (3),

$$
\begin{aligned}
\frac{\partial u_{i}}{\partial t}+\left(\bar{U}_{k}+u_{k}\right) \frac{\partial u_{i}}{\partial x_{k}} & =-\frac{1}{\rho} \frac{\partial p}{\partial x_{i}}+v \frac{\partial^{2} u_{i}}{\partial x_{k} \partial x_{k}} \\
-2 \varepsilon_{i k l} \Omega_{k} u_{l} & +f\left(v_{i}-u_{i}\right)
\end{aligned}
$$

For an incompressible fluid $\left(u_{i} \frac{\partial u_{k}}{\partial x_{k}}\right)_{A}=0$ so that Equation (6) can be written as

$$
\begin{aligned}
\frac{\partial}{\partial t}\left(u_{i}\right)_{A}+ & {\left[\bar{U}_{k}+\left(u_{k}\right)_{A}\right]\left(\frac{\partial}{\partial x_{k}}\right)_{A}\left(u_{i}\right)_{A}+\left(u_{i} \frac{\partial u_{k}}{\partial x_{k}}\right)_{A} } \\
=- & \frac{1}{\rho}\left(\frac{\partial}{\partial x_{i}}\right)_{A} p_{A}+v\left(\frac{\partial^{2}}{\partial x_{k} \partial x_{k}}\right)_{A}\left(u_{i}\right)_{A} \\
& -2\left(\varepsilon_{i k l} \Omega_{k} u_{l}\right)_{A}+f\left(v_{i}-u_{i}\right)_{A}
\end{aligned}
$$

$$
\begin{gathered}
\left(u_{j}\right)_{B} \frac{\partial}{\partial t}\left(u_{i}\right)_{A}+\left[\bar{U}_{k}+\left(u_{k}\right)_{A}\right]\left(\frac{\partial}{\partial x_{k}}\right)_{A}\left(u_{i}\right)_{A}\left(u_{j}\right)_{B} \\
+\left(u_{i}\right)_{A}\left(\frac{\partial}{\partial x_{k}}\right)_{A}\left(u_{k}\right)_{A}\left(u_{j}\right)_{B}=-\frac{1}{\rho}\left(\frac{\partial}{\partial x_{i}}\right)_{A} p_{A}\left(u_{j}\right)_{B} \\
+v\left(\frac{\partial^{2}}{\partial x_{k} \partial x_{k}}\right)_{A}\left(u_{i}\right)_{A}\left(u_{j}\right)_{B}-2\left(\varepsilon_{i k l} \Omega_{k} u_{l}\right)_{A}\left(u_{j}\right)_{B} \\
+f\left(v_{i}-u_{i}\right)_{A}\left(u_{j}\right)_{B}
\end{gathered}
$$

Where $\left(u_{j}\right)_{B}$ can be treated as a constant in a differential process at the point $A$.

Similarly, the energy equation for $u_{j}$ at the point $B$ is obtained as

$$
\begin{gathered}
\frac{\partial u_{j}}{\partial t}+\left(\bar{U}_{k}+u_{k}\right) \frac{\partial u_{j}}{\partial x_{k}}=-\frac{1}{\rho} \frac{\partial p}{\partial x_{j}}+v \frac{\partial^{2} u_{j}}{\partial x_{k} \partial x_{k}} \\
-2 \varepsilon_{j k l} \Omega_{k} u_{l}+f\left(v_{j}-u_{j}\right)
\end{gathered}
$$

Since for an incompressible fluid $\left(u_{j} \frac{\partial u_{k}}{\partial x_{k}}\right)_{B}=0$ then the above equation can be written as

$$
\begin{aligned}
\frac{\partial}{\partial t}\left(u_{j}\right)_{B}+ & {\left[\bar{U}_{k}+\left(u_{k}\right)_{B}\right]\left(\frac{\partial}{\partial x_{k}}\right)_{B}\left(u_{j}\right)_{B}+\left(u_{j} \frac{\partial u_{k}}{\partial x_{k}}\right)_{B} } \\
= & -\frac{1}{\rho}\left(\frac{\partial}{\partial x_{j}}\right)_{B} p_{B}+v\left(\frac{\partial^{2}}{\partial x_{k} \partial x_{k}}\right)_{B}\left(u_{j}\right)_{B} \\
& -2\left(\varepsilon_{j k l} \Omega_{k} u_{l}\right)_{B}+f\left(v_{j}-u_{j}\right)_{B}
\end{aligned}
$$

Multiplying Equation (9) by $\left(u_{i}\right)_{A}$, we get

$$
\begin{gathered}
\left(u_{i}\right)_{A} \frac{\partial}{\partial t}\left(u_{j}\right)_{B}+\left[\bar{U}_{k}+\left(u_{k}\right)_{B}\right]\left(\frac{\partial}{\partial x_{k}}\right)_{B}\left(u_{j}\right)_{B}\left(u_{i}\right)_{A} \\
+\left(u_{j}\right)_{B}\left(\frac{\partial}{\partial x_{k}}\right)_{B}\left(u_{k}\right)_{B}\left(u_{i}\right)_{A}=-\frac{1}{\rho}\left(\frac{\partial}{\partial x_{j}}\right)_{B} p_{B}\left(u_{i}\right)_{A} \\
+v\left(\frac{\partial^{2}}{\partial x_{k} \partial x_{k}}\right)_{B}\left(u_{j}\right)_{B}\left(u_{i}\right)_{A}-2\left(\varepsilon_{j k l} \Omega_{k} u_{l}\right)_{B}\left(u_{i}\right)_{A} \\
+f\left(v_{j}-u_{j}\right)_{B}\left(u_{i}\right)_{A}
\end{gathered}
$$

Where $\left(u_{i}\right)_{A}$ can be treated as a constant in a differential process at the point $B$.

Addition of Equation (8) and Equation (10) gives the result 


$$
\begin{gathered}
\frac{\partial}{\partial t}\left(u_{i}\right)_{A}\left(u_{j}\right)_{B}+\left[\left(\frac{\partial}{\partial x_{k}}\right)_{A}\left(u_{i}\right)_{A}\left(u_{k}\right)_{A}\left(u_{j}\right)_{B}+\left(\frac{\partial}{\partial x_{k}}\right)_{B}\left(u_{i}\right)_{A}\left(u_{k}\right)_{B}\left(u_{j}\right)_{B}\right] \\
+\bar{U}_{k}\left[\left(\frac{\partial}{\partial x_{k}}\right)_{A}\left(u_{i}\right)_{A}\left(u_{j}\right)_{B}+\left(\frac{\partial}{\partial x_{k}}\right)_{B}\left(u_{i}\right)_{A}\left(u_{j}\right)_{B}\right] \\
=-\frac{1}{\rho}\left[\left(\frac{\partial}{\partial x_{i}}\right)_{A} p_{A}\left(u_{j}\right)_{B}+\left(\frac{\partial}{\partial x_{j}}\right)_{B} p_{B}\left(u_{i}\right)_{A}\right] \\
+v\left[\left(\frac{\partial^{2}}{\partial x_{k} \partial x_{k}}\right)_{A}+\left(\frac{\partial^{2}}{\partial x_{k} \partial x_{k}}\right)_{B}\right]\left(u_{i}\right)_{A}\left(u_{j}\right)_{B}-2\left(\varepsilon_{i k l} \Omega_{k} u_{l}\right)_{A}\left(u_{j}\right)_{B} \\
\left.+\left(\varepsilon_{j k l} \Omega_{k} u_{l}\right)_{B}\left(u_{i}\right)_{A}\right]+f\left[\left(v_{i}-u_{i}\right)_{A}\left(u_{j}\right)_{B}+\left(v_{j}-u_{j}\right)_{B}\left(u_{i}\right)_{A}\right]
\end{gathered}
$$

To expose the relation of turbulent energy in presence of dust particles at the point $B$ to those at point $A$, it will give no difference if we take one point as the origin of $A$ or $B$ of the coordinate system.

Let us consider the point $A$ as the origin. In order to differentiate between the effects of distance and location, we introduce as new independent variables,

$$
\zeta_{k}=\left(x_{k}\right)_{B}-\left(x_{k}\right)_{A}
$$

Then we obtain,

$$
\begin{aligned}
& \left(\frac{\partial}{\partial x_{k}}\right)_{A}=-\frac{\partial}{\partial \zeta_{k}},\left(\frac{\partial}{\partial x_{k}}\right)_{B}=\frac{\partial}{\partial \zeta_{k}} \\
& \left(\frac{\partial^{2}}{\partial x_{k} \partial x_{k}}\right)_{A}=\left(\frac{\partial^{2}}{\partial x_{k} \partial x_{k}}\right)_{B}=\frac{\partial^{2}}{\partial \zeta_{k} \partial \zeta_{k}} .
\end{aligned}
$$

Using the above relations and taking ensemble average on both sides, Equation (11) becomes

$$
\begin{aligned}
& \frac{\partial}{\partial t} \overline{\left(u_{i}\right)_{A}\left(u_{j}\right)_{B}}-\frac{\partial}{\partial \zeta_{k}} \overline{\left(u_{i}\right)_{A}\left(u_{k}\right)_{A}\left(u_{j}\right)_{B}}+\frac{\partial}{\partial \zeta_{k}} \overline{\left(u_{i}\right)_{A}\left(u_{k}\right)_{B}\left(u_{j}\right)_{B}} \\
& =\frac{1}{\rho}\left[\frac{\partial}{\partial \zeta_{i}} \overline{p_{A}\left(u_{j}\right)_{B}}+\frac{\partial}{\partial \zeta_{j}} \overline{p_{B}\left(u_{i}\right)}\right]+2 v \frac{\partial^{2}}{\partial \zeta_{k} \partial \zeta_{k}} \\
& \overline{\left(u_{i}\right)_{A}\left(u_{j}\right)_{B}}-2\left[\overline{\left(\varepsilon_{i k l} \Omega_{k} u_{l}\right)_{A}\left(u_{j}\right)_{B}}+\overline{\left(\varepsilon_{j k l} \Omega_{k} u_{l}\right)_{B}\left(u_{i}\right)_{A}}\right] \\
& +f\left[\overline{\left(v_{i}\right)_{A}\left(u_{j}\right)_{B}}-2 \overline{\left(u_{i}\right)_{A}\left(u_{j}\right)_{B}}+\overline{\left(u_{i}\right)_{A}\left(v_{j}\right)_{B}}\right]
\end{aligned}
$$

Equation (12) represents the mean motion for turbulent energy in presence of dust particles and pressure-velocity correlation.

It is noted that the coefficient of $\bar{U}_{k}$ has been vanished. The Equation (12) describes the turbulent energy motion in presence of dust particles, where the motions with respect to a coordinate system moving with the mean velocity $\bar{U}_{k}$. Equation (12) contains the double velocity correlation $\overline{\left(u_{i}\right)_{A}\left(u_{j}\right)_{B}}$, double correlations such as $p_{A}\left(u_{j}\right)_{B}$, triple correlations such as $\left(u_{i}\right)_{A}\left(u_{k}\right)_{A}\left(u_{j}\right)_{B}$ where all the terms apart from one another. The correlations $\overline{p_{A}\left(u_{j}\right)_{B}}$ and $\overline{p_{B}\left(u_{i}\right)}$ form the tensors of first order, because pressure is a scalar quantity and the triple correlations $\overline{\left(u_{i}\right)_{A}\left(u_{k}\right)_{A}\left(u_{j}\right)_{B}}$ and $\overline{\left(u_{i}\right)_{A}\left(u_{k}\right)_{B}\left(u_{j}\right)_{B}}$ form the tensors of third order. The double and triple correlations at the two points $A$ and $B$ in the flow field have been shown in Figure 1 and Figure 2 respectively, where $r$ is the distance between two points $A$ and $B$.

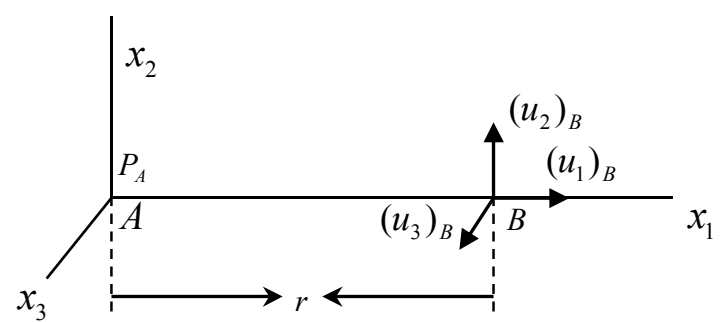

Figure 1(a). Double correlation between pressure at $A$ and velocity components at $B$.

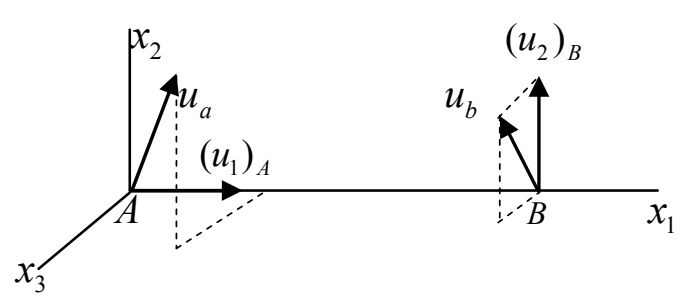

Figure1(b). Double velocity correlation between the velocities $u_{a}$ at $A$ and $u_{b}$ at $B$.

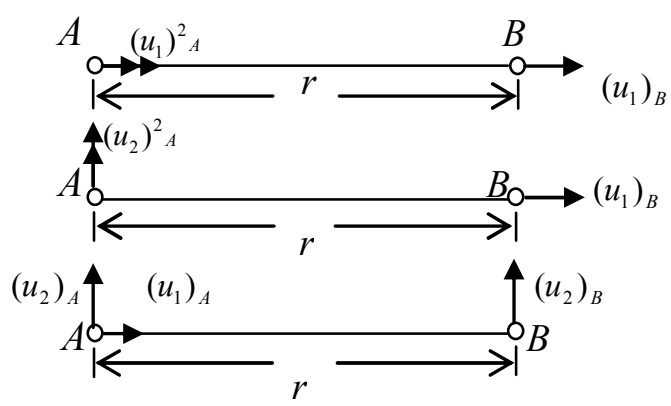

Figure 2. Triple velocity correlation among the velocities at the points $A$ and $B$.

We designate the first order correlations by $\left(k_{p, j}\right)_{A, B}$, second order correlations by $\left(Q_{i, j}\right)_{A, B}$ and third order correlations by $\left(s_{i k, j}\right)_{A, B}$. Therefore, we set 


$$
\begin{aligned}
& \left(k_{i, p}\right)_{A, B}={\overline{\left(u_{i}\right)_{A} p_{B}}}_{,}\left(k_{p, j}\right)_{A, B}=\overline{p_{A}\left(u_{j}\right)_{B}}, \\
& \left(Q_{i, j}\right)_{A, B}=\overline{\left(u_{i}\right)_{A}\left(u_{j}\right)_{B},}
\end{aligned}
$$

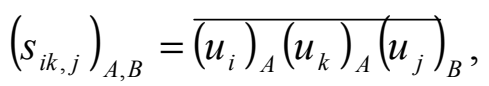

$$
\begin{aligned}
& \left(s_{i, k j}\right)_{A, B}=\overline{\left(u_{i}\right)_{A}\left(u_{k}\right)_{B}\left(u_{j}\right)_{B}}, \\
& \left(F_{i, j}\right)_{A, B}=\overline{\left(v_{i}\right)_{A}\left(u_{j}\right)_{B}} \text {, and }\left(G_{i, j}\right)_{A, B}=\overline{\left(u_{i}\right)_{A}\left(v_{j}\right)_{B} .}
\end{aligned}
$$

Where, the index $p$ indicates the pressure and is not a dummy index like $i$ or $j$ so that the summation convention does not apply to $p$.

Also the terms $\left(\varepsilon_{i k l} \Omega_{k} u_{l}\right)_{A}\left(u_{j}\right)_{B} \quad$ and $\left(\varepsilon_{j k l} \Omega_{k} u_{l}\right)_{B}\left(u_{i}\right)_{A}$ form the correlation tensors of second order, we designate these by $D_{i, j}$ and $H_{i, j}$ respectively.

Thus we set

$$
\left(D_{i, j}\right)_{A, B}=\overline{\left(\varepsilon_{i k l} \Omega_{k} u_{l}\right)_{A}\left(u_{j}\right)_{B}}
$$

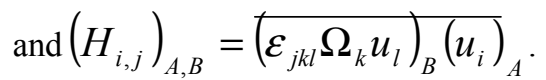

If we use the above relations of first, second and third order correlations in Equation (12) then we obtain

$$
\begin{gathered}
\frac{\partial}{\partial t} Q_{i, j}-\frac{\partial}{\partial \zeta_{k}} S_{i k, j}+\frac{\partial}{\partial \zeta_{k}} S_{i, k j}=-\frac{1}{\rho}\left(\frac{\partial}{\partial \zeta_{i}} K_{p, j}+\frac{\partial}{\partial \zeta_{j}} K_{i, p}\right) \\
+2 v \frac{\partial^{2}}{\partial \zeta_{k} \partial \zeta_{k}} Q_{i, j}-2\left[\left(D_{i, j}+H_{i, j}\right)\right]+f\left(F_{i, j}-2 Q_{i, j}+G_{i, j}\right)
\end{gathered}
$$

Where all the correlations refer to the two points $A$ and $B$.

Now for an isotropic turbulence of an incompressible flow, the double pressure-velocity correlations are zero, that is,

$$
\left(k_{p, j}\right)_{A, B}=0,\left(k_{i, p}\right)_{A, B}=0 .
$$

In case of isotropy, the statistical features have no directional preference and perfect disorder persists. The velocity fluctuations are independent of the axis of reference, i.e. invariant to axis rotation and reflection.

From the definition of isotropy, $\left(Q_{i, j}\right)_{A, B}=\overline{\left(u_{i}\right)_{A}\left(u_{j}\right)_{B}}=0$ for all $i \neq j$. In the rotating system in the flow field through $180^{\circ}$ about $x_{1}$-axis must, because of isotropy, give

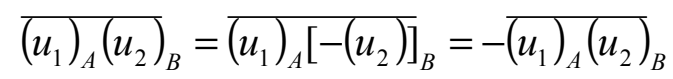

which can be true only when $\overline{\left(u_{1}\right)_{A}\left(u_{2}\right)_{B}}=0$.

The isotropic turbulence in a bounded domain is a model where in the turbulence is unaffected by the boundaries enclosing the fluid, and furthermore the statistical moments are spatially invariant and independent of orientation. Isotropic grid turbulence is a similar idealization, in that the turbulence is enclosed by wind tunnel walls and the homogeneity of the turbulence in the central region is known to be unaffected by the wall boundary layers. In an isotropic turbulence it follows from the condition of invariance under reflection with respect to point $A$,

$$
\overline{\left(u_{i}\right)_{A}\left(u_{k}\right)_{B}\left(u_{j}\right)_{B}}=-\overline{\left(u_{k}\right)_{A}\left(u_{j}\right)_{A}\left(u_{i}\right)_{B}}
$$

or, $\left(s_{i, k j}\right)_{A, B}=-\left(s_{k j, i}\right)_{A, B}$

In absence of isotropic turbulence, physical properties will be different in different directions according to the direction of measurement. Anisotropic turbulence tends toward local isotropy, in that the statistics of velocity differences tend toward invariance under rotation as the distance between the velocities becomes smaller. For non-isotropic (anisotropic) turbulence, constant or non-constant average velocity of pressure field will not be zero. Anisotropy is the property of being directionally dependent. It can be defined as a difference, when measured along different axes, in a material's physical or mechanical properties (absorbance, refractive index, conductivity, tensile strength, etc.).

Thus Equation (13) can be written as

$$
\begin{gathered}
\frac{\partial}{\partial t} Q_{i, j}-\frac{\partial}{\partial \zeta_{k}}\left(S_{i k, j}+S_{k j, i}\right)=2 v \frac{\partial^{2}}{\partial \zeta_{k} \partial \zeta_{k}} Q_{i, j} \\
-2\left[\left(D_{i, j}+H_{i, j}\right)\right]+f\left(F_{i, j}-2 Q_{i, j}+G_{i, j}\right)
\end{gathered}
$$

The term $\frac{\partial}{\partial \zeta_{k}}\left(S_{i k, j}+S_{k j, i}\right)$ forms the tensor of second order; we designate these by $S_{i, j}$. Also, the terms $\left(D_{i, j}+H_{i, j}\right)$, we designate this by $L_{i, j}$.

Thus we set,

$$
S_{i, j}=\frac{\partial}{\partial \zeta_{k}}\left(S_{i k, j}+S_{k j, i}\right) \text {, and } L_{i, j}=\left(D_{i, j}+H_{i, j}\right) .
$$

Therefore Equation (14) gives the result

$$
\begin{aligned}
\frac{\partial}{\partial t} Q_{i, j} & -S_{i, j}=2 v \frac{\partial^{2}}{\partial \zeta_{k} \partial \zeta_{k}} Q_{i, j}-2 L_{i, j} \\
& +f\left(F_{i, j}-2 Q_{i, j}+G_{i, j}\right)
\end{aligned}
$$

Equation (15) is the energy equation for dusty fluid 
turbulent flow in terms of correlation tensor of second order.

In absence of dust particles, $f=0$, then Equation (15) reduces to

$$
\frac{\partial}{\partial t} Q_{i, j}-S_{i, j}=2 v \frac{\partial^{2}}{\partial \zeta_{k} \partial \zeta_{k}} Q_{i, j}-2 L_{i, j}
$$

Equation (16) describes the energy motion for turbulent flow in terms of the correlation tensor of second order.

If there are no effects of the dissipation $\varepsilon$ by the turbulence per unit mass, $L_{i, j}=0$ so that the Equation (16) takes the form

$$
\frac{\partial}{\partial t} Q_{i, j}-S_{i, j}=2 v \frac{\partial^{2}}{\partial \zeta_{k} \partial \zeta_{k}} Q_{i, j}
$$

Equation (17) represents the turbulent motion in terms of correlation tensors of second order which is the same as obtained Hinze [7].

\section{Results and Discussion}

The resulting Equation (15) has been developed for dusty fluid turbulent energy in terms of second order correlation tensor. Thus the Equation (15) represents the energy equation of turbulent motion in terms of correlation tensors of second order in presence of dust particles. In this equation, all the terms $Q_{i, j}, S_{i, j}, F_{i, j}, G_{i, j}, L_{i, j}$ are the second order correlation tensors where, $Q_{i, j}$ and $S_{i, j}$ represents the velocity correlations at the two points $A$ and $B$ of the flow field, $F_{i, j}$ and $G_{i, j}$ represents velocity correlations between the fluid velocity components and solid particles (dust) velocity components at the two points whereas $L_{i, j}$ represents the correlation between turbulent energy and velocity of the fluid particles. An interaction between the dust particles through collisions and through the effects on the flow of the fluid is shaped in the neighborhood of the particles. But in absence of the dissipation $\mathcal{E}$ by the turbulence per unit mass and without any effect of dust particles to the fluid velocity, the resulting Equation (15) reduces to the Equation (17) which represents the turbulent motion.

\section{Conclusion}

The dust particles into a turbulent carrier flow decrease the level of turbulence of the gas phase because of particles involvement into fluctuation motion. The amplitude of turbulent fluctuations of particle velocity in the axial direction is much higher than that in the radial direction. Connection of the turbulent flow and particle motions can be described in different ways, when considering particles in a flow. One-way coupling is the simplest approach, where the particles follow the flow due to the forces acting on the particles, i.e. the particles are light. The second is the two-way coupling, where forces are exerted in both directions, i.e. the fluid act on the particle and the particle acts back on the fluid, e.g. due to wakes behind particles. In a three-way coupling, in addition to what was previously mentioned, the particles interact with each other through hydrodynamic forces; this is of increased importance for larger concentrations.

In presence of dust particles, the equation of motion of turbulent energy has been derived by averaging procedure, which includes the effect of dust particles and the correlations between the pressure fluctuations and velocity fluctuations at two points of the flow field. If there is no effect of dust particles in the flow field, the resulting Equation (15) reduces to Equation (16) which represents the energy motion for turbulent flow in terms of the correlation tensor of second order and in absence of the dissipation $\mathcal{E}$ by the turbulence per unit mass the Equation (16) reduces to Equation (17) which gives the turbulent motion in terms of correlation tensors of second order that is the same as obtained Hinze (1975).

\section{References}

[1] Ahmed, S.F., (2013). Derivation of Turbulent Energy in a Rotating System. Journal of Computational and Applied Research in Mechanical Engineering, 3(1): pp. 75-83.

[2] Ahmed, S.F. and Sarker, M.S.A., (2011). Fiber suspensions in turbulent flow with two-point correlation. Bangladesh Journal of Scientific and Industrial Research, 46(2): pp. 265-270.

[3] Bosse, T. and Kleiser, L., (2006). Small particles in homogeneous turbulence: Settling velocity enhancement by two-way coupling. Physics of Fluids, 18: pp. 027102-17.

[4] Bracco, A., Chavanis, P.H. and Provenzale, A., (1999). Particle aggregation in a turbulent Keplerian flow. Physics of Fluids, 11(8): pp. 2280-2287.

[5] Chandrasekhar, S., (1955). Hydro magnetic turbulence. II. An elementary theory. Proceedings of the Royal Society, 233: pp. 330-350.

[6] Crowe, C.T., Troutt, T.R. and Chung, J.N., (1996). Numerical models for two-phase turbulent flows. Annual Review of Fluid Mechanics, 28: pp.11-43.

[7] Havnes, O. and Kassa, M., (2009). On the sizes and observable effects of dust particles in polar mesospheric winter echoes. Journal of Geophysical Research, DOI: 10.1029/2008JD011276, 114: D09209.

[8] Hinze, J.O., (1975). Turbulence, New York: McGraw-Hill Book Company.

[9] Hodgson, L.S. and Brandenburg, A. 1998. Turbulence effects in planetesimal formation. Astronomy and Astrophysics. 330: 1169-1174.

[10] Hussainov, M., Kartushinsky, A., Rudi, U., Shcheglov, I., Kohnen, G. and Sommerfeld M., (2000). Experimental investigation of turbulence modulation by solid particles in a 
grid-generated vertical flow. International Journal of Heat and Fluid Flow, 21: pp. 365-373.

[11] Johansen, A., Henning,T. and Klahr, H., (2006). Dust sedimentation and self-sustained kelvin-helmholtz turbulence in protoplanetary disk midplanes. The Astrophysical Journal, 643: pp. 1219-1232.

[12] Kishore, N. and Sarker, M.S.A., (1990). Rate of change of vorticity covariance in MHD turbulent flow of dusty incompressible fluid. International Journal of Energy Research, 14(5): pp. 573-577.

[13] Kvasnak, W., Ahmadi, G., Bayer, R. and Gaynes, M., (1993). Experimental investigation of dust particle deposition in a turbulent channel flow. Journal of Aerosol Science, 24: pp. 795-815.

[14] Nickovic, S., Kallos G., Papadopoulos, A. and Kakaliagou, O., (2001). A model for prediction of desert dust cycle in the atmosphere. Journal of Geophysical Research, 106: pp. 18113-18129.

[15] Oakey, N.S., (1982). Determination of the rate of dissipation of turbulent energy from simultaneous temperature and velocity shear microstructure measurements. Journal of
Physical Oceanography, 12: pp. 256-271.

[16] Okuzumi, S. and Hirose, S., (2011). Modeling magnetorotational turbulence in protoplanetary disks with dead zones. The Astrophysical Journal, DOI:10.1088/0004-637X/742/2/65, 742: 65 .

[17] Pakhomov, M.A., Protasov, M.V., Terekhov, V.I. and Varaksin, A.Y., (2007). Experimental and numerical investigation of downward gas-dispersed turbulent pipe flow. International Journal of Heat and Mass Transfer, 50: pp. $2107-2116$

[18] Pan, L., Padoan, P., Scalo, J., Kritsuk, A.G. and Norman, M.L., (2011). Turbulent clustering of protoplanetary dust and planetesimal formation. The Astrophysical Journal, DOI: 10.1088/0004-637X/740/1/6, 740(1):6.

[19] Saito, M.B. and Lemos, M.J.S.de., (2010). A macroscopic two-energy equation model for turbulent flow and heat transfer in highly porous media. International Journal of Heat and Mass Transfer, 35 (11-12): pp. 2424-2433.

[20] Sarker, M.S.A and Ahmed, S.F., (2011). Fiber motion in dusty fluid turbulent flow with two-point correlation. Journal of Scientific Research, 3 (2): pp. 283-290. 